\title{
LA LÓGICA DEL CUIDADO EN ESTRATEGIAS SANITARIAS DESTINADAS A LA POBLACIÓN MATERNO INFANTIL ${ }^{1}$ THE LOGIC OF CARE IN SANITARY STRATEGIES AIMED AT MATERNAL CHILD POPULATION
}

RESUMEN: Se trata de una investigación etnográfica donde se analizan las acciones sanitarias destinadas a la población materno infantil desde la perspectiva del cuidado. Objetivos: a) Identificar las prácticas y discursos que despliegan los agentes del sistema de salud oficial para el tratamiento de déficits nutricionales, b) Explorar las percepciones de la población paciente sobre la atención médica del sistema de salud oficial, c) Identificar prácticas de cuidado locales. Metodología: cualitativa, observación participante, entrevistas en profundidad y técnicas de documentación en sectores rurales del departamento de Orán, Salta, Argentina. Resultados: Las prácticas de APS se comprenden en el marco de estudios de gobernabilidad y se sustentan en el modelo de familia nuclear. Las madres constituyen pacientes activas que elaboran estrategias para el cuidado de la salud de sus hijos. Emergen mediaciones entre el saber biomédico y prácticas médicas populares en las relaciones que establecen los agentes sanitarios y sus pacientes. Conclusiones: La noción de cuidado, resulta valiosa para develar el entramado de desigualdades en que se construye la vulnerabilidad de las poblaciones destinatarias de las políticas de APS. Los pacientes constituyen sujetos activos que buscan instancias terapeúticas donde se registre las sensaciones de sus cuerpos y se considere su subjetividad.

Palabras claves: etnografía; salud; cuidado; género; desnutrición.

\footnotetext{
${ }^{1}$ Este artículo es una reformulación de la ponencia presentada en la $\mathrm{X}^{\mathrm{a}}$ Reunión de Antropología del Mercosur (RAM), en la ciudad de Córdoba, Argentina, Julio 2013. Agradezco a las críticas y comentarios recibidos en la mesa coordinada por Zulmira Newland Borges y Cynthia Fortuna del Rio. ${ }^{2}$ Doctoranda en Antropología Social por el Consejo Nacional de Investigaciones Científicas y Técnicas - CONICET. Profesora en Antropología Social de la Facultad de Filosofía y Letras de la Universidad de Buenos Aires - UBA, Buenos Aires, D.F. Argentina. E-mail: pialeavy@gmail.com 
ABSTRACT: This article is based on ethnographic research where sanitary actions destined to maternal and child populations are analyzed in the frame of care studies. Objectives: a) Identify discourses and practices used by agents of official health care system for nutritional deficits treatments, b) Explore patient population perceptions regarding official medical attention system, c) Identify local health care practices. Methodology: qualitative, participant observation, deep interviews and documentation techniques in rural regions in Oran department, Salta, Argentina. Results: PHC practices are included under governability studies framework and sustained in nuclear family patterns. Mothers are active patients who elaborate strategies for their children health care. From biomedical knowledge and traditional health practices, mediations emerge between sanitary agents and their patients. Conclusions: health care idea is critical to reveal the inequality spider web in which PHC politics addressed population vulnerability is built. Patients are active subjects who search for therapeutic instances where their body sensations are record and their subjectivity is respected.

Keywords: Ethnography; health; gender; care; nutritional deficit.

\section{INTRODUCCIÓN}

La implementación de políticas públicas constituye una interacción entre instituciones, acciones y actores que contribuye al "desarrollo de la gobernabilidad de las vidas en las sociedades contemporáneas" (Herkovits, 2007, p.204). En relación a las políticas sociosanitarias, dicha racionalidad de gobierno promociona una serie de ideales regulatorios articuladas con complejas tecnologías para la conducción de la conducta, que se sustentan en una determinada concepción de sujeto. La misma se estructura en torno a la autonomía, la capacidad de elección, la libertad, la identidad y la eficiencia, entendiendo que "la persona es un agente activo, deseoso de ejercer una responsabilidad informada, autónoma y secular sobre su propio destino" (Lorenzetti, 2007, p.2). Esta dinámica de gobierno, propia del liberalismo avanzado, configura intervenciones sociales que suponen 
un sujeto autónomo que logra superar las limitaciones de su entorno de acuerdo a las elecciones y acciones que realiza (Rose, 2003).

De acuerdo con Mol (2008), esta concepción de sujeto se enmarca en la "lógica de la elección" que organiza modos de actuar, de concebir los cuerpos, las emociones y los comportamientos de los sujetos, además de proveer una determinada moralidad. En esta lógica, la biomedicina construye parámetros e indicadores sobre los estilos de vida de los pacientes, para que sean conocidas y mejoradas por ellos mismos con la finalidad de mejorar, controlar e impedir la enfermedad (Epele, 2007). En contraposición al paradigma de la elección, Mol (2008) explica la "lógica del cuidado", que se sustenta en la necesidad intrínseca de los seres humanos de vincularse y de asumir responsabilidades para cuidar unos de otros ${ }^{3}$. Examinar las prácticas de cuidado, ilumina temas devaluados por las sociedades contemporáneas, como la vulnerabilidad y la dependencia de todos los sujetos y su necesidad de ser cuidados. Si analizamos "quienes cuidan", se evidencian todos los patrones de subordinación que organizan la sociedad y cómo aquellos que cuidan - las clases bajas, las mujeres, las minorías étnicas- deben cuidarse a si mismos (Tronto, 1993 apud, Epele, 2010, p.2). De este modo, la "racionalidad del cuidado" permite problematizar toda la serie de valores que sustentan las políticas públicas liberales tensionando las oposiciones entre autonomía y dependencia, control y atención, entre otras (Mol, 2008).

Las prácticas del "buen cuidado" -good care- (op.cit, 2008) implican el despliegue de saberes y técnicas que ayudan a un persona a sentirse mejor en la experiencia del padecimento. Para conseguir el bienestar del otro, el cuidador debe considerar su subjetividad, sus emociones y su cuerpo, debe atender a una respuesta. Así pues, la acción de cuidar, contiene una lógica procesual, se acciona a partir de la advertencia de una necesidad, implica asumir una responsabilidad con otro, involucra una actividad física, la realización de una acción y al mismo tiempo su respuesta (Tronto, 1993).

Aunque la "necesidad de ser cuidados" constituya una condición

${ }^{3}$ El hecho de que la especie humana tenga crías que nacen a los nueve meses, con un período fetal de veintiún meses, ubica a la misma como la "prole más frágil y dependiente de todos los mamíferos" (Hernando, 2008, p.66). 
humana para la existencia, lo que se entiende por cuidado, está sujeto a un tiempo y un espacio determinado. Los grupos sociales identifican ciertas prácticas y técnicas como cuidado y descartan otras (Epele, 2011). En este sentido, el enfoque antropológico aporta herramientas útiles para indagar qué prácticas médicas pueden comprenderse dentro de la lógica del cuidado, que impliquen el compromiso con la subjetividad del paciente y el registro de su cuerpo, entre otras cuestiones.

En este artículo utilizaremos los lineamientos teóricos de los estudios sobre el cuidado en salud (Tronto, 1993; Mol, 2008), para reflexionar en torno a las acciones que se realizan desde el Programa de Atención Primaria de la Salud (PROAPS) hacia la población paciente. En este caso particular, nos enfocamos en el modo en que se construye la población objetivo de las políticas sanitarias que buscan disminuir los niveles de déficit nutricionales en sectores del Chaco Salteño argentino. Partimos de la hipótesis de que atendiendo a las prácticas de "buen cuidado" (Tronto, 1993) podremos conocer limitaciones del sistema de salud oficial. A partir de la labor etnográfica, se plantean los objetivos de identificar los discursos y prácticas que se realizan desde el sector médico sobre las madres de niños con déficit nutricional; explorar las percepciones de la población paciente sobre la atención médica del sistema de salud oficial e identificar prácticas de cuidado locales.

\section{EL CUIDADO DE LA SALUD MATERNO-INFANTIL}

Si bien la crianza de los hijos es una actividad que caracteriza supuestamente a todos los grupos domésticos en todas las sociedades humanas, los modos en que los sujetos se organizan en torno a esta actividad son construidos culturalmente (Goody, 1994). En las sociedades occidentales, con el advenimiento de la modernidad y la separación de las esferas institucionales, especialmente las productivas de las económicas, determinados patrones sociales comienzan a organizar la división social del trabajo entre los miembros de "la familia nuclear" (Jelín, 1998, p.45). Este modelo de familia plantea que existen expectativas sociales que distinguen el trabajo de los hombres y el de las mujeres. Los hombres son los responsables de 
los insumos económicos que se consiguen afuera del hogar mientras las mujeres se encargan de las actividades domésticas "puertas adentro". Así pues, la edad constituye un factor organizador, porque define a los ancianos como dependientes y a los niños como seres inherentemente frágiles y carentes de autonomía (Szulc, 2006). En correspondencia con la "familia patriarcal" (Jelín, 1998), en el modelo "nuclear" el hombre posee el rol de autoridad como jefe de familia y la mujer es responsable de las tareas reproductivas, tales como gestar, tener hijos y responsabilizarse del mantenimiento y la subsistencia del resto de los integrantes de la familia. Entre estas tareas, las mujeres también son responsables de:

"La reproducción social, en las tareas destinadas al mantenimiento del sistema social, especialmente en el cuidado y la socialización temprana de los niños y niñas, transmitiendo normas y patrones de conducta aceptados y esperados" (Jelín, 1998, p.46).

De este modo, las actividades humanas referidas al cuidado infantil han estado históricamente relegadas al ámbito privado doméstico, en oposición al ámbito público (Moore, 1988). Se entiende que el ámbito privado es el de los afectos y los sentimientos, que las madres protegen con el "poder del amor" (Jelín, 1998). La moralidad que promueve este modelo de familia, postula que la maternidad es un "don natural" y que aquellas madres que no muestran dedicación a sus hijos son incapaces o indignas y sus comportamientos pueden ser calificados como patológicos (Marcus, 2003).

El despliegue de toda una serie de saberes científicos que dictaminen los modos correctos de "ser madre" y "de crecer", fue necesario para legitimar los postulados vinculados al modelo de "familia nuclear”. En este sentido, Badinter (1982) analiza cómo los estudios científicos promovieron a la construcción del instinto maternal, el amor espontáneo, inmutable e incondicional que surge de toda mujer hacia sus hijos creando la obligación de ser madres. El "amor maternal" que aparece en el siglo XVIII como algo natural que obliga a las madres a garantizar la educación de sus hijos, representa una matriz 
de significados, prácticas y sentimientos que son social, histórica y culturalmente producidos (Tarducci, 2008; Palomar Verea, 2005). Cuestiones como la lactancia materna, se tornan argumentos claves para la legitimidad de una crianza, ya que constituye el componente básico de la correcta nutrición del niño y responsable del vínculo indisoluble entre los dos (Nari, 2000).

En paralelo con la construcción social del instinto maternal, la infancia es distinguida como ciclo vital y adquiere una nueva serie de valores (Ariés, 1962). Las nociones de crecimiento y desarrollo constituyen el "núcleo definitorio de la condición infantil" (Colángelo, 2008, p.3) que no sólo justifican su abordaje médico específico sino también la intervención de toda una serie de profesionales que dictaminan aquello que es esperable o no de una conducta infantil para la concreción de una vida socialmente aceptada. Asimismo, el amor maternal, entendido como una aptitud natural, se promueve como factor clave desde el saber pediátrico para asegurar el desarrollo del niño.

Las representaciones sobre la niñez y la maternidad integran procesos biológicos y culturales, constituyendo un nexo primario entre lo público y lo privado y creando espacios susceptibles de ser intervenidos por actores y saberes, que por ejemplo, transmiten determinados modos de criar a un niño y no otros (Scheperd-Hughes y Sargent, 1998). La niñez emerge entonces como un terreno en disputa donde intervienen distintos actores e instituciones entre las que se encuentra la familia y el estado. Ante la moralidad que se establece sobre los modos correctos de crianza de los niños y niñas, nos preguntamos: ¿qué grado de autonomía tienen las madres para decidir como quieren criar a sus hijxs?, ¿qué saberes emergen como legítimos para cuidar un niño y cualés no?; ¿cómo operan sobre las madres los saberes legitimados sobre el cuidado infantil?, ¿qué prácticas médicas pueden entenderse dentro de la "lógica del cuidado" y cuáles no?

\section{METODOLOGÍA Y ÁREA DE INVESTIGACIÓN}

El trabajo de campo se realizó entre noviembre de 2010 y marzo de 2013 en sectores rurales del Departamento de Orán, Salta, 
Argentina. Se definió como campo de trabajo el paraje " 26 ", donde habitan familias de origen criollo, ava-guaraní y kolla dentro y fuera de terrenos privados destinados a la explotación agrícola. El acceso al campo se realizó a través de la participación en el equipo interdisciplinario de investigación sobre parasitosis en zonas endémicas a cargo del Instituto de Investigaciones de Enfermedades Tropicales (IIET) de la Universidad Nacional de Salta (UNSA) (Socías et. al, 2012).

La metodología se nutrió de las técnicas cualitativas de observación participante, entrevistas abiertas y semiestructuradas, el fichado bibliográfico y el análisis de datos secundarios, como investigaciones desde las ciencias sociales, documentos y estudios médicos utilizados por el PROAPS 5 . Los datos se registraron mediante un grabador digital, notas de campo y una cámara de fotos. La observación participante se realizó acompañando a lxs agentes sanitarios en sus visitas domiciliarias y en sus actividades de formación y seguimiento, a las madres en las actividades laborales agrícolas, en las tareas domésticas en sus hogares y en los espacios comunes de las fincas, en las salas de salud rurales y en el hospital regional. Los objetivos que guiaron la investigación fueron indagar identificar las prácticas y discursos que despliegan los agentes del sistema de salud oficial para el tratamiento de déficits nutricionales, explorar las percepciones de la población paciente sobre la atención médica del sistema de salud oficial e identificar prácticas de cuidado locales. En todo el trabajo se preserva la identidad de lxs sujetos del campo a través del uso de seudónimos para las personas y los lugares.

EL " 26 " se ubica en la cuenca del Alto Bermejo, que constituye uno de los hábitats de la selva pedemontana, también conocida como yungas o selvas de altura. Las condiciones ambientales del clima subtropical de las yungas, junto con el proceso de distribución de la tierras en la provincia de Salta (Buliubasich y Rodríguez, 2009; Rodríguez García, 2011) han convertido a la zona en un importante polo económico de trabajo agrícola, al cual han migrado histórica-

\footnotetext{
${ }^{4}$ Nombre ficticio.

${ }^{5}$ El PROAPS funciona en la provincia de Salta desde 1978 y es el programa nacional que nuclea y organiza las acciones del primer nivel de atención a la salud, también conocidas como la Atención Primaria de la Salud (APS). Si bien depende del gobierno nacional y provincial, su financiamiento proviene de entidades como el Programa de Naciones Unidas para el Desarrollo (PNUD) o el Banco Mundial.
} 
mente obrerxs bolivianxs y argentinxs de distintas etnias. Históricamente, esta región agro-industrial se constituyó a partir de la explotación maderera, la producción de azúcar, a manos del ingenio azucarero Tabacal, las empresas agropecuarias -fincas- que demandan mano de obra intermitente para su producción y la explotación de hidrocarburos. En la última década, el avance de la tecnología agrícola -especialmente el paquete maíz-soja transgénico- generó el avance de la frontera cultivable y el desmonte de tierras de las yungas. Estos procesos generaron que la pauperización de las poblaciones criollas, kollas y ava-guaraníes locales (Lorenzetti, 2013).

La vida al margen de las tierras productivas de la población trabajadora (Rodríguez García, 2009) junto con sus bajos indicadores sociales $^{6}$, conforma el marco ideal para la endemicidad de distintas enfermedades tropicales (Gil et al, 2011). Las familias donde no hay trabajadores empleados formalmente reciben Asignación Universal por Hijo ${ }^{7}$. La atención médica de estos grupos sociales, está a cargo del PROAPS, que depende del Ministerio Provincial de Salta. Organizadxs territorialmente, lxs agentes sanitarios del PROAPS focalizan su atención médica en la población materno-infantil, visitando principalmente las viviendas donde habitan menores de seis años y embarazadas. En el "26" se emplaza uno de los Centros de Atención Primaria de la Salud (CAPS) además de un arroyo y un grupo de casas donde viven las familias kollas, ava-guaraníes y criollas. El nexo entre la población afectada y las intervenciones en salud, públicas o privadas, son los agentes sanitarios del PROAPS.

\footnotetext{
${ }^{6}$ En el 2001 el 43,2\% de la población de Orán contaba con Necesidades Básicas Insatisfechas, superando en un $10 \%$ la media provincial. Actualmente no poseemos datos que confirmen este indicador, pero sabemos que de los 31.859 hogares de dicho departamento, el 40,2\% no posee desagües cloacales. Ver http://www.censo2010.indec.gov.ar/CuadrosDefinitivos/H2-D_66_126. pdf. Acceso 25/4/2014.

${ }^{7}$ Es un beneficio que le corresponde a lxs hijos de las personas desocupadas, que trabajan en el mercado informal o que ganan menos del salario mínimo, vital y móvil. Consiste en el pago mensual de \$460 para niños menores de 18 años y de $\$ 1500$ para chicos discapacitados sin límite de edad. Con la misma, el Estado busca asegurarse de que los niños y adolescentes asistan a la escuela, se realicen controles periódicos de salud y cumplan con el calendario de vacunación obligatorio, ya que éstos son requisitos indispensables para cobrarla (1/11/2009, decreto 1602/09). Disponible: http://www.anses.gob.ar/destacados/asignacion-universal-por-hijo-1. Acceso 24/9/2013.
} 


\section{RESULTADOS Y DISCUSIÓN DE LA LABOR ETNOGRÁFICA}

\subsection{Lxs agentes de la salud}

Los postulados de la Atención Primaria de la Salud (APS) son, principalmente, trabajar con las causas y no con las consecuencias de las enfermedades incluyendo como fundamentos contribuir al desarrollo local, la participación comunitaria y el trabajo intersectorial, a través de la figura central del agente sanitario como promotor/a de actividades de promoción y prevención de la salud (Torres Aliaga y Torres Secchi, 2011). Entre los objetivos principales de APS se encuentran ser la entrada al sistema de salud; asegurar una atención permanente y longitudinal a la persona, aún en ausencia de enfermedad y proveer una atención integral que haga frente a todas las necesidades de las personas (Maceira, 2007).

Lxs agentes sanitarixs deben identificar y clasificar los problemas de salud sobre la base del análisis epidemiológico de la morbi-mortalidad y dar respuesta inmediata para su solución en el nivel de entrada o para su referencia a niveles de mayor complejidad. Así pues, una de las actividades principales de su trabajo es "salir a censar" ", es decir, a contar y describir lxs sujetos que conforman su territorio -sector de salud- a trabajar. La actividad del censo se realiza con los formularios del PROAPS, que sistematizan descripciones tanto del hogar como de las características de lxs integrantes de la familia. Las descripciones se fundamentan en paradigmas epidemiológicos, que apuntan a medir las condiciones de vida de los pacientes en términos de "exposición a riesgos a la salud". Una vez que se marcan los riesgos, éstos son sumados siguiendo puntajes y marcados en cada formulario. Las familias con puntajes más altos -las que acumulan más tipos de riesgos- serán aquellas donde los agentes focalizarán su atención. Observando la sistematización de riesgos, la

\footnotetext{
${ }^{8}$ Las frases encodilladas en cursiva pertenecen a notas de campo.

${ }^{9}$ Los factores de riesgo son: "Grupo integrado por menores de 6 años"; "Desempleo y/o ingreso económico inestable (trabajo irregular, jornalero)"; "Ausencia de Padre o Madre (Padre o Madre soltero/a- Viudo/a)"; "Carencia de Obra Social"; "Enfermedades crónicas en la familia"; "Enfermedades Sociales"; "Niños menores de 6 años con déficit nutricional"; "Embarazada"; "Tratamiento Inadecuado de Agua, Residuos"; "Analfabetismo de la persona a cargo de los menores"; "Mortalidad Infantil/ Materna"; "Recién nacido/Puérpera" (Formulario $N^{\circ} 1$ ).
} 
existencia de niñxs en el hogar, sanos o no, tanto como embarazadas, es un factor para definir la prioridad de la atención.

En cada visita lxs agentes deben "educar" a lxs integrantes de su sector de salud, "fomentando hábitos adecuados de la salud" y "detectando hábitos de riesgo" por medio de discursos. "Ustedes deben ser como hormiguitas que se meten en las casas y en la cabeza de sus pacientes, deben ver todo y luego avisarnos, porque sólo así sabremos como actuar" expresa en una charla para agentes sanitarios una pediatra a cargo de una de las áreas de APS del Departamento de Orán. El trabajo de hormiguita implica la permanente observación y transmisión de información hacia el equipo médico. De este modo se imprime un carácter especial en la relación médicxs/agentes, donde lxs primerxs poseen el saber científico y lxs segundxs son lxs que traen la información del campo que sirve para diseñar acciones. "Ustedes son los captadores y los salvadores de vida, ustedes son los que observan bien a los chiquitos y a las madres" continúa la médica, subrayando la importancia de la atención a estos grupos sociales.

Más allá de la función pedagógica de la metáfora del trabajo del agente sanitario igualado al de una hormiga, esta idea de que estos empleados de la salud pública deben meterse en la cabeza de los pacientes se relaciona con los lineamientos de los estudios de gobernabilidad (Gordon, 1991; Rose, 2003). Estos estudios comprenden que los agentes estatales gobiernan a partir de complejas tecnologías que operan sobre la conducta de los sujetos. En términos de políticas sanitarias, es a través de la promoción de ideales regulatorios tales como los hábitos saludables - dieta adecuada, mantenimiento de higiene personal- y la responsabilidad que las personas evitan y previenen la emergencia de enfermedades. Lorenzetti (2011; 2007) utiliza los estudios de gobernabilidad para explicar las acciones de lxs agentes sanitarios en zonas aledañas del chaco salteño, argumentando que la relación entre lxs pacientes y lxs agentes sanitarios cobra un carácter especial: 
"En términos generales, quienes trabajan en APS realizan una primera distinción que establece una demarcación entre el agente sanitario como aquel que 'enseña', y, la población asignada como la que debe ser 'promovida'. De este modo, los lugares donde se trabaja son caracterizados como sectores ‘carentes' o 'faltos de', otorgándole a la relación agente- familia un carácter particular”. (Lorenzetti, 2007, p.10)

La particularidad de esta relación reside en que lxs agentes explican hábitos correctos a sujetos que son entendidxs como autónomxs, responsables y también vulnerables y "carentes”. Así mismo, el rol del agente sanitario puede implicar tensiones, porque ellos mismos son originarios de las comunidades en las que atienden, por lo tanto, al mismo tiempo que son la "cara visible del Estado" son parte “de la población objetivo" (Op.cit, p.13).

Esta trama de tensiones entre saberes científicos-legítimos/ elegítimos, vulnerabilidad/autonomía y “carencias sociales"/responsabilidad se observa también en las recomendaciones que se dan desde el equipo médico para el cuidado de la salud infantil.

\subsection{La identificación de madres "en déficit"}

Los déficits nutricionales se presentan como un emergente de las condiciones de vida de la población infantil, que se construye como la población en riesgo por excelencia, por la demanda de nutrientes que necesita para su correcto crecimiento (Torres, 2012). En Argentina no hay un sistema de vigilancia nutricional que permita monitorear la situación, pero por las encuestas permanentes realizadas por el Ministerio de Salud, la desnutrición aguda se mueve en niveles poco importantes a nivel poblacional ${ }^{10}$. El problema grave pasa entonces por la desnutrición crónica, responsable de que "los niños con déficit en su alimentación no alcancen la altura que deberían tener a su edad $\mathrm{y}$, a nivel poblacional, indiquen una situación socio-económica desfavorable" (Aguirre, 2010, p.92). Ante los casos de desnutrición aguda y crónica, en Salta se implementaron programas de asistencia nutricio-

${ }^{10}$ Perfil Salud de la Niñez Ambiental en Argentina, Ministerio de Salud de la Nación, 2007. 
nal para madres y menores de seis años, que incluyen el suministro de leche fortificada para niños menores de dos años ${ }^{11}$.

Si bien en el departamento de Orán, el PROAPS llega a cubrir el $60 \%$ de la población total, los niveles de desnutrición infantil alcanzan el 9\% en zonas operativas como la de San Francisco ${ }^{12}$. Por este motivo, gran parte de las tareas de los agentes sanitarios se destinan actualmente al tratamiento de déficits nutricionales de la población infantil. En las pre-rondas, se encuentran lxs agentes de una zona operativa, para sistematizar los datos obtenidos en los censos, compartirlo con sus supervisores y recibir charlas de los integrantes de los equipos médicos de la institución médica de referencia donde se definen las acciones a futuro. En una de estas charlas, una médica con cargo gerencial explicaba a los agentes cómo debían manejarse ante un posible caso de desnutrición y/o deshidratación ${ }^{13}$ :

"Ustedes deben observar, preguntar y explorar, si el chico juega o no, cómo tiene la elasticidad de la piel, como respira. Un niño desnutrido es un changol4 que sufre, que no tiene presencia en el ambiente, si no juega o corre es que algo anda mal. Es un tema dificil porque podemos ver a la mamá gorda con el chiquito flaco, pero bueno, no se confien en la madre, ella puede estar bien alimentada pero el bebé no". (Pediatra a cargo de APS, Pre-ronda, 3/9/2012)

Cabe destacar que el exceso de peso de la madre no implica “buena alimentación”. Diversos estudios analizan los complejos procesos sociales y económicos involucrados en el acceso a alimentos de los sectores populares y la calidad de los mismos (ver Aguirre 2010, 2004; Ortale, 2003). Ante el encarecimiento de los productos

\footnotetext{
11 Disponible en http://www.salta.gov.ar/prensa/noticias/en-salta-la-lucha-contra-la-desnutricion-es-politica-de-estado/25788. Acceso 14/3/2014.

12 Datos PROAPS, Pre ronda Diciembre 2011, Orán, Salta. La desnutrición aguda infantil, tiene poca importancia epidemiológica en Argentina(3\%), mientras la desnutrición crónica , a la cual se refieren los programas de políticas públicas, es prevalente y se manifiesta en los niveles bajos de talla y peso y su secuela más común es el déficit de hierro (Ortale,2003). La provincia de Salta junto con Formosa, tiene la tasa más alta de mortalidad por desnutrición de menores de 5 años de 1,9 y 1,7 por mil nacidos vivos, respectivamente (Ministerio de Salud de la Nación. Dirección Nacional de Estadísticas e Información, 2011).

${ }^{13}$ La deshidratación existe cuando un cuerpo no posee el líquido suficiente para llevar a cabo sus funciones básicas. Sus síntomas son diarrea, vómitos, sudor y falta de apetito entre otros.

${ }^{14}$ Niño.
} 
alimentarios y la consecuente inaccesibilidad, los sectores de menor poder adquisitivo eligen alimentos "rendidores", es decir, aquellos que sean baratos, llenen y gusten. De este modo, las dietas de estos hogares se componen de alimentos de mayor sensación de saciedad, tales como fideos, papas, carnes grasas y azúcares, por lo tanto saturadas de hidratos y grasas. Las consecuencias se advierten en los cuerpos más gruesos por el exceso de grasas e hidratos ricos en energía y más bajos que el resto de la población, porque no llegan a desarrollar el potencial genético de altura debido a la desnutrición crónica (Calvo, et al. 2001). Las madres del 26 no consumen las frutas y verduras que cosechan porque no tienen acceso a los mismos y su contextura física gorda no implica calidad nutricional.

Sin embargo, desde la mirada de algunos médicos y agentes sanitarios, la mama gorda con chiquito flaco emerge como una contradicción que simboliza grados de negligencia materna. En las instrucciones a lxs agentes sanitarios hay una determinada indicación de cómo proceder, que descarga moralmente contra la madre en caso de que exista un caso niñx con déficit nutricional. Se entiende que la actitud irresponsable de la madre posibilita la emergencia del/a niñx con déficit. De este modo, la mala conducta de la madre emerge como el agente etiológico del cuadro clínico, que sustenta una actitud del equipo médico: la desconfianza y la sospecha sobre la población paciente de madres.

“Qué es lo que más le preocupa a las madres en un cuadro de diarrea? Que gasta más pañales para el hijo, que todo el día debe cambiarlos, entonces a veces ni los cambian y los dejan así."(Pediatra Cardiólogo, pre-ronda 4/9/2012)

"No se confien en lo que dicen, la clínica es soberana, esto quiere decir que deben mirar al nene y a la madre, deben mirar, no escuchar porque pueden engañar"'(Pediatra, charla pre-ronda, 3/9/2012) "Las madres tienen seis, siete hasta ocho hijos quizás, todos dando vueltas sin que se los pueda criar bien, tienen que insistir en que presten más atención a los hijos, ellas no lo hacen" (Supervisor APS área rural,charla preronda, 4/9/2012) 
En estas tramas discursivas se configuran las prácticas de intervención sobre la población materno infantil. La actitud inadecuada de las madres está constituida por el descuido y la desidia en la crianza de sus hijxs, como consecuencia de la falta de planificación familiar. La figura masculina en la crianza emerge por omisión, no se exige nada a los varones padres, sino a las madres. En este sentido, el "ojo clínico" no es neutro, se fundamenta en ciertas expectativas de familia que definen modos adecuados de crianza. A las madres se las debe mirar más que escuchar. Tiene más legitimidad la "clínica" entendida como la mirada y observación de los síntomas de los pacientes. Entonces, la autonomía de los sujetos pacientes está dada por su capacidad de tomar decisiones (in)correctas sobre su salud o la de sus descendientes, pero no así por su capacidad de expresar sensaciones o percepciones sobre su cuerpo o el de sus hijxs.

Entre las actitudes que desde el equipo médico se califican como incorrectas del lado de las madres, se encuentra la de suministrar al bebé algún tipo de remedio casero para tratar los episodios de deshidratación generados por diarrea.

"Cualquier tipo de té de yuyos que vean en la mamadera o que vean en la cocinita de las madres no duden si pueden en tirarlo, eso es veneno, ¡Veneno! ¿Escucharon? Nos cansamos de decir que los remedios caseros no sirven y hasta pueden hacer peor, así que hay que terminar con ellos"(Charla Pre-Ronda, pediatra a cargo de área de APS, 3/9/2012).

Las infusiones elaboradas con plantas autóctonas -hojas de payco o poleo "burrito" hervidas- y otro tipo de recetas naturales -semillas de papaya molidas con leche- son utilizadas con fines terapéuticos por varios habitantes del 26. Dependiendo del origen étnico de lxs sujetos, se despliegan toda una serie de prácticas en relación al cuidado del cuerpo y el tratamiento de malestares. Las mismas son rechazadas por algunxs representantes del sistema de salud oficial, pero utilizadas y permitidas por otrxs. Nos interesa indagar en las madres que utilizan este tipo de recetas, como las conceptualizan y que situaciones las lleva a utilizar este tipo de recetas o prácticas de curación. 


\subsection{La perspectiva de las madres}

Rosa tiene 36 años, se identifica como criolla y vive en el 26 desde que cumplió los catorce. Trabajó en todas las fincas de la zona, hasta que su marido fue designado encargado de una y el patrón les dio un galpón de herramientas para que lo utilicen de vivienda. En esta misma construcción Rosa vive con sus dos hijxs menores y su pareja. Para el abastecimiento de agua llenan tanques de 200 litros con la bomba de la finca donde trabaja el hijo, las traen en tractor hasta la entrada de su casa y las cubren con plástico para evitar que "se llenen de bichos15". Al frente de la casa, Rosa construyó una despensa donde vende bebidas y comidas para empleados de fincas de la zona y así aporta económicamente a la subsistencia del hogar, "con esta edad ya no puedo más ir al campo a trabajar" explica. Para obtener mercadería a precio más económico, Rosa viaja a la feria de alimentos de Bermejo (Bolivia) dos veces por semana.

El cuidado de la salud de sus hijxs siempre fue complicado en la zona: "Acá hay de todo, estamos llenos de bichos y sin agua, los chicos se pescan todo, yo he sabido tener mucho 'desarreglo' con mis hijos". El desarreglo es el modo local de denominar cualquier malestar estomacal, más precisamente la diarrea, tanto en niños como en adultos. Rosa coincide con las estadísticas del PROAPS ${ }^{16}$ en que durante el verano, la diarrea es uno de los problemas que más afecta a la población infantil y cuenta su experiencia con una de sus hijas:

"Mi Laura de changuita17 estaba muy mal. Flaquita, flaquita, la diarrea no le cortaba y era como un bebé sin vida, tenia un año pero yo creía que la perdía. Yo la llevaba al médico, y el médico siempre siempre con lo mismo, dándome manzana rallada y agua de arroz, diciéndome que ya iba a pasar. Ya va a pasar me decía. Yo estaba desesperada. [...] le pedía al patrón algo de plata para el micro a

\footnotetext{
${ }^{15}$ Los bichos pueden ser insectos como los mosquitos o parásitos, como el Ascaris Lumbricoides que genera patologías gastrointestinales.

${ }^{16}$ Según información suministrada por agentes sanitarios, durante la época estival los padecimientos infantiles más frecuentes son las patologías gastrointestinales y las deshidrataciones, mientras que en el invierno son protagonistas las infecciones respiratorias.

${ }^{17}$ De pequeña.
} 
la banda18 y ahí nomás llevé a mi hija a un tipoy19 que me habian recomendado. Un viejito que se quedaba con la niña en una cama y la fumateaba20 toda, le decía su nombre al oído. Yo le pagaba con zapallitos o pimientos, no tenía mas para darle. La llevé como tres veces y luego la niña empezó a estar bien [...] Yo nunca se lo dije ni al agente ni al médico. Y mira si yo me quedaba con la manzanita rallada, ;mi hija se me moría!. Es como que si uno no se mueve no pasa nada. Y uno tiene que probar, uno por sus hijos prueba todo. Y yo creo, yo creo, yo creo, aquel que no crea no se si le sale bien y con el curandero mi hija se curó. El curandero estaba como una hora cada vez que íbamos, la miraba mucho, la oía respirar, yo acá si voy al médico siento que ni nos miran, ni nos prestan atención cuando vamos a la consulta"(13/03/2011)

Según Menéndez (2004) las personas que sufren un padecimiento demandan inicialmente un tipo de atención en función del diagnóstico presuntivo que manejan y según otros factores como la accesibilidad física y económica a diversas formas de atención que operan en su contexto de vida. Para Rosa lo más accesible al principio era visitar al médico, pero cuando esta atención le resultó ineficaz, buscó la atención de otro tipo de curador y/o instancia terapeútica. En este sentido, su origen criollo no la excluye de buscar modos de atención pertenecientes a las etnias guaraníes. En la búsqueda del bienestar de su hija, Rosa realiza una articulación práctico-ideológica de distintas formas de atención, no rechaza el saber biomédico al visitar al curandero, sino que se apropian de ambos saberes, para ver y sentir cuál de ellos les da herramientas o recetas más eficaces para que su hija se sienta mejor.

En su experiencia visitando curanderos, Rosa cuenta una serie de elementos que diferencian a este espacio terapeútico de la consulta en la sala de salud:"cuando vamos al médico de la salita, es como si no nos prestara atención, algunos a veces ni te habla, ni

18 Se denomina "La banda" a las ciudades fronterizas, en este caso Rosa se refiere a la ciudad boliviana de Bermejo.

19 En "El Bananal" se les dice tipoy a los curanderos guaraníes. El tipoy es una prenda, una túnica que cubre todo el cuerpo, utilizada prioritariamente por mujeres, pero también por hombres. Es un símbolo de la etnia guaraní que se adapta luego de la colonización. (Albó,1995)

20 Fumatear consiste en exhalar humos sobre la persona. 
te miran a los ojos". En cambio, la curandera "miraba y oía" a su hija. Esta escucha, implica una atención a las sensaciones de la persona que está atravesando el padecimiento. La visita a la curandera implicó también un vínculo con ella y una respuesta. Rosa remarca que no se siente "ni mirada" cuando visita al médico de la sala de salud, a pesar de que se atiende con médicos del sistema oficial que salud que enfatizan la necesidad de mirar y observar a lxs pacientes en las visitas de lxs agentes sanitarios. En este sentido, Mol (2008) explica que en las consultas con un médico, los pacientes emergen como objetos pasivos, que no "tienen ni la oportunidad de hablar por si mismos"(2008, p.6, traducción propia). Por su parte, la atención en la lógica del cuidado, el proceso de atención y tratamiento considera la subjetividad y los saberes del paciente, por lo tanto importa la respuesta y el vínculo que se construye con el cuidador.

Epele (1997) analiza el modelo de consulta médica en el cual el médico es el que posee el saber/poder y el paciente debe adecuarse a tratamientos que indican pautas de auto-cuidado. Para aquellas personas que sufren padecimientos crónicos y conviven con enfermedades, las pautas de auto-cuidado implican situaciones conflictivas entre atención, cuidado y control de si mismo, porque el paciente debe realizar ciertas prácticas para mantener su estado de salud, a pesar no sentir una respuesta favorable. En este sentido, Rosa explica que si no tenía la iniciativa de consultar por fuera del sistema médico oficial no había solución al problema de salud de su hija: "Acá es como que si una no se encarga, nadie lo hará, si yo me quedaba sentada con la receta del médico y la visita del agente no conseguía nada. Acá si no es por uno mismo, no se consigue nada". Rosa marca la importancia de la autonomía propia, pero no en un sentido positivo, sino como una sensación de abandono ante la cual debe emprender una estrategia. Desde su discurso, la autonomía tiene un sentido de denuncia frente al auto -cuidado que le exige el sistema de salud.

\section{4 "Líneas de fuga" entre agentes y pacientes}

Las relaciones entre lxs agentes sanitarios y las personas pa- 
cientes se dan "no sólo en términos de causalidad y oposición, sino como relaciones que pueden contener líneas de fuga, haciendo de la divergencia y la distancia otras relaciones posibles"(Viveiros de Castro, 2007 citado en Bonet y Gomez Tavare, 2010, p.197, traducción propia). No siempre los pacientes ocultan sus visitas al curandero o el suministro de remedios caseros, ni siempre lxs agentes sanitarios rechazan estas prácticas.

Nancy es una madre kolla de 28 años, también pareja de un encargado de otra de las fincas del Bananal. Ellxs viven ocho meses en el 26 y cuatro meses en la ciudad boliviana de Tarija. Tienen dos hijos, uno de dos años y otro de cuatro años. Además de trabajar en el hogar, ella vende golosinas y bebidas en su casa para el resto de familias de la finca. Al igual que Rosa, dos veces por semana cruza a la banda a buscar mercadería, porque son más baratas en Bolivia, "como los remedios y los curanderos". La vida en este espacio de frontera, permite la continua circulación de personas, saberes, mercancías - tales como medicamentos- y servicios, que varían en función de los valores de las monedas de ambas naciones.

En una visita domiciliaria con Carmen, una agente sanitaria, conocí a Nancy y sus hijos. Mientras atendía su pensión, Nancy nos contaba un episodio que había sufrido su hijo en el verano:

"Mi hijo Cristian tenía mucha diarrea y no se detenía. Me habian dicho que eran los parásitos, y bueno, no se que era, pero mucha diarrea y el estaba todo amarillito y débil. Tomó el desparasitante y no pasaba nada, seguía mal, yo estaba muy preocupada y cuando nos fuimos a Bolivia, mi hermana me mandó a una curandera que me dio unos yuyos para hacer un té, que debía dárselos un par de veces al día"(17/03/2011)

Nancy cuenta la historia al lado de la agente sanitaria que no realiza ninguna objeción. Cuando salimos de su casa le pregunté a la agente por la visita a las curanderos y ella me explicó que ella también a veces iba y que toda su familia lo hacía. Si bien esta misma agente también estaba en las reuniones de instrucciones para el "tratamiento 
con madres", no le dijo a la madre que los remedios eran "veneno". Recordamos el planteo de Lorenzetti (2008) de que los agentes sanitarios ocupan un lugar de tensión con la comunidad, y agregamos el rol activo de los mismos, que deciden qué decir a la población, más allá de las directivas que tengan. De este modo, la tarea de promover hábitos de salud es elaborada en la experiencia de cada agente sanitario y en el vínculo con los pacientes de su sector de salud.

En este sentido, podemos abrir un campo de exploración sobre las mediaciones (Bonet y Gomes Tavares, 2010) que elaboran las personas en sus trayectorias y acciones dentro del sistema de salud. Si bien todo el marco referencial en que los agentes realizan sus acciones se sustenta en criterios epistemológicos sobre los que se fundamenta el saber biomédico (Good, 1994) ellos viven en el mismo contexto que sus pacientes, por lo tanto poseen todo un acervo de conocimientos en común que les permite compartir representaciones y saberes sobre el proceso de salud/enfermedad/atención. En este sentido, la forma en que un grupo articula su "saber médico con el saber de otros sectores" es lo que posibilita entender "su tipo de saber, que no se define en términos de tradicional o moderno ni de científico/no científico"(Menéndez, 1994, p.76).

Volviendo a la acción específica de la curandera, Nancy relata que los yuyos que le había recetado la curandera eran infusiones de manzanilla. Pensando en la calidad del agua que se consume en la zona, la modalidad de recetar un té, puede ser beneficiosa porque implica hervir el agua, que no siempre está en buen estado para consumo. Pero nos interesa rescatar el modo en que Nancy relata la consulta con la curandera: "Ella es como una abuelita, que acariciaba mucho al niño cada vez que el iba, y lo tuve que llevar varias veces hasta que vio que estaba bien".

Las acciones de "la abuelita" implican un registro de la actividad del cuerpo del paciente, una observación de su respuesta y una consideración de emociones y sensaciones, tanto de la madre como del bebé. Son estos elementos que tanto Rosa como Nancy remarcan como fundamentales para la instancia terapéutica y ausentes en la consulta médica. Desde el lado del/a cuidadora o curador/a, ellas 
rescatan la existencia de un registro de la subjetividad del otro, en contraposición a los médicos de la sala que "ni las miran". De acuerdo con Mol (2008) esta dinámica en las consultas con un médico, que construye a los pacientes como objetos pasivos, debe ser problematizada. Por su parte, Epele (1997) argumenta que la modalidad de tratamiento de las patologías en el sistema biomédico, se centraliza en los aspectos biológicos, orgánicos y fisiológicos de la salud/enfermedad, postergando tanto los aspectos subjetivos como los procesos socio-culturales implicados en el mismo. Los aspectos subjetivos, tales como las sensaciones, emociones y percepciones del pacientes, son el eje del "buen cuidado". Nancy y Rosa no rechazaron la atención brindada por el médico local, pero sólo encontraron eficacia en el curandero. Ellas destacan la labor de éste último, no sólo porque le brindó bienestar a sus hijos, sino también porque en el tratamiento recibido observaron una dinámica distinta a la experimentada en la consulta de la sala de salud, donde se percibía y se atendía detenidamente la respuesta de sus hijxs. En estas cuestiones observamos qué prácticas se conciben como "buen cuidado" localmente.

Así pues, el cuidado materno-infantil, si bien constituye una de las categorías conceptuales y discursivas más utilizadas en la promoción de políticas públicas destinadas a la infancia, en términos prácticos permanece relegado al ámbito de lo privado y lo doméstico. En tanto la familia nuclear continúe siendo el modelo para el diseño de políticas públicas (Cosse,2003; Nari,2000), se restringe el compromiso masculino en la crianza al plano de lo económico, responsabilizando a las madres por el crecimiento, desarrollo y cuidado de los hijxs. Por su parte, la lógica de elección en las políticas sanitarias, opaca los procesos históricos sociales y políticos conformadores de las condiciones de vida de las poblaciones vulnerables y que posibilitan, la emergencia de déficit nutricionales.

\subsection{A modo de cierre}

A lo largo del trabajo, hemos analizado el problema de los déficit nutricionales en poblaciones vulnerables del Chaco-Salteño, obser- 
vando las construcciones sobre la maternidad y el cuidado infantil que poseen los efectores del sistema de salud oficial y la perspectiva de las madres sobre la atención médica infantil. Sostenemos que si bien las relaciones entre los efectores del sistema de salud y los pacientes no son lineales, las acciones que buscan la prevención y el tratamiento de patologías en el crecimiento y el desarrollo infantil, se basan un modelo de familia nuclear (Jelín,1998) que ubica a las madres en el lugar de máximas responsables ante los padecimiento de los más pequeños. La emergencia de déficits nutricionales es un complejo problema de salud pública, que se comprende a través del análisis del acceso a alimentos de los sectores populares, la desigual distribución de la tierra y del acceso a recursos naturales (ver Aguirre, 2007).

La noción de cuidado, resulta valiosa para develar el entramado de desigualdades en que se construye la vulnerabilidad de las poblaciones destinatarias de las políticas de APS. En el trabajo de campo, he presenciado cómo se les niega a los trabajadores agrícolas el acceso a las frutas y verduras que cosechan. Las madres trabajadoras, "gordas con chiquitos flacos" que "pueden engañar" y que "no prestan atención a sus hijos", cosechan los alimentos que otros sectores sociales eligen comer para cuidar su salud a través de una dieta balanceada.

El "ideal de elección" (Mol,2008) en el marco del PROAPS se expresa en la promoción de pautas de auto-cuidado. Los médicos y los agentes sanitarios recomiendan los modos ideales de vivir, prescribiendo pautas de cómo ser madre, cúantos hijos tener y cómo hervir el agua, sin prestar atención a cómo los pacientes lo hacen, ni cómo cuidan de su salud. La "lógica del cuidado" en este contexto nos permite ver lo que los sujetos hacen para buscar el bienestar de sus familiares, por fuera del sistema oficial de salud, en un espacio donde se registre las sensaciones de sus cuerpos y se registre la respuesta a las acciones del cuidador. El significado de la supuesta autonomía de lxs sujetos pacientes, emerge como una cuestión a problematizar, ya que emerge como una cualidad ponderada desde el ideal de elección pero falsa e inútil en la búsqueda de instancias terapeúticas.

La vulnerabilidad emerge como otra de las categorías a problematizar desde la perspectiva antropológica. La emergencia de ca- 
sos de desnutrición aguda en el contexto de la presente investigación, ha generado una serie de explicaciones desde distintos efectores de salud y funcionarios estatales que responsabilizan que argumentan la emergencia de dicha problemática por las características culturales de los pacientes ${ }^{21}$. Como antropólogxs del campo de la salud, debemos advertir sobre los efectos de estos "argumentos culturalistas" (Lorenzetti, 2011) que responsabilizan la vulnerabilidad de las poblaciones en características culturales o las particularidades del clima subtropical. Este tipo de argumentos dan lugar a una esencialización de las ideas de clase social, pertenencia étnica y vulnerabilidad que evita dar cuenta de los procesos conformadores de la vulnerabilidad de ciertos sectores sociales (Frankenberg et al.2003). Esta idea de vulnerabilidad, centrada en 1xs individuos, no sólo opaca procesos sociales, sino que también fundamenta la responsabilidad la población paciente sobre los padecimientos que sufre.

\section{REFERENCIAS}

AGUIRRE, P. Ricos flacos y gordos pobres. La alimentación en crisis. Buenos Aires: Editorial Capital Intelectual, 2010.

. Pobreza y Globalización en la emergente epidemia de obesidad (traducción). In: BROWN, F. (Comp.). Diabetes in Women. Boston Mass.: Humana Press, 2007.

- Estrategias de consumo: ¿qué comen los argentinos que comen? Buenos Aires: Ed. Miño y Dávila, 2004.

ARIES, P. Centuries of Childhood. London: Cape, 1962.

BADINTER, E. ¿Existe el instinto maternal? Historia del amor maternal siglos XXVII al XX. Barcelona: Paidós, 1991.

BULIUBASICH, C. y RODRIGUEZ, H. La noción de trabajo en la construcción de la identidad: indígenas y criollos en el Pilcomayo salteño. Cuad. antropol. soc. [online]. 2002, nº.16 [citado 2012-0806], pp. 185-209 . Disponible en: <http://www.scielo.org.ar/scielo. php?script=sci_arttext\&pid=S1850-275X2002000200004\&lng=es

\footnotetext{
${ }^{21}$ Según Urtubey los aborígenes desnutridos no van al hospital por "una cuestión cultural". Disponible en http://www.lapoliticaonline.com/nota/nota-71200. Acceso 20/4/2014.
} 
$\& n r m=$ iso $>$. ISSN $1850-275 X$.

CAlVO, E.; LONGO, E.; AGUIRRE, P.; BRITOS, S. Prevenciones de la anemia de niños y embarazadas en la Argentina. Actualización para equipos de salud. Dirección Nacional de Salud Materno Infanto Juvenil. Junio de 2001. Disponible en http:/www.msal.gov.ar/ promin/publicaciones/pdf/15-anemia.pdf. Acceso: 26/3/2014.

CASTILLA, V. y LORENZO, G. Emociones en suspenso: marginalidad y consumo de pasta base/paco en barrios marginales de Buenos Aires. Cuadernos de Antropología Social, n.36, pp.69-89, 2012.

COLÁNGELO, A. La Constitución de la niñez como objeto de estudio de intervención de los médicos en la Argentina de comienzos del siglo XX. IX $X^{\circ}$ Congreso Argentino de Antropología Social: Fronteras de la Antropología, 5 al 8 de agosto de 2008, Misiones, Argentina.

COSSE, I. Estigmas de nacimiento. Peronismo y orden familiar. Buenos Aires: FCE, 2006.

EPELE, M. Emergencies and Rescues: the Logics of Vulnerability and Care among Drug Users in Buenos Aires, Argentina. Addiction Research and Theory. [En prensa], 2011.

. Sujetar por la herida. Buenos Aires: Paidós. 2010, Cap. 10.

. La lógica de la Sospecha. Sobre Criminalización del Uso de Drogas, Complots y Barreras de Acceso al Sistema de Salud. Cuadernos de Antropología Social, no 25, pp.151-168, 2007.

FRANKENBERG, R. Unidos por la diferencia, divididos por la semejanza: la alegremente dolorosa posibilidad de colaboración entre medicina y antropología. Cuadernos de Antropología Social, nº.17, pp.11-27, 2003.

FRANKENBERG, R.; ROBINSON, I.; DELAHOOKE, A. Countering essentialism in behavioural social science: the example of 'the vulnerable child' ethnographically examinated. Sociological Review, Vol.48, n4, pp.586-611, 2000.

GIL, J.; CIMINO, R.; LOPEZ QUIROGA, I.; CAJAL, P.; ACOSTA, N.; JUAREZ, M.; ORELLANA, V.; KROLIEWIECKI A.; DIOSQUE, P.; NASSER, J. Reactividad del antígeno GST-SAPA de Trypanosoma cruzi frente a sueros de pacientes con enfermedad de Chagas y leishmaniasis. Medicina, Buenos Aires, Vol.71, $\mathrm{n}^{\mathrm{o}} .2$, pp.113-119, 2011. 
GORDON, C. Govermental rationality: an introduction. In: BURCHELL, G.; GORDON, C.; MILLER, P. (Eds.). The Foucault effects. Studies in Governmentality. Chicago: The University of Chicago Press, 1991.

GRINBERG, M. De conceptos y métodos: relaciones entre epidemiología y antropología. In: ÁlVAREZ, M. y BARREDA, V. (Comp.). Cultura, Salud y Enfermedad. Temas en Antropología Médica. Buenos Aires: Instituto Nacional de Antropología y Pensamiento Latinoamericano, 1997, pp.11-23.

GOOD, B. J. How Medicine Constructs its Objetcs. In: GOOD, B. J. Medicine, Rationality, and Experience. An Anthropological Perspective. Cambridge: Cambridge University Press,1994, pp.6587.

HERKOVITS, D. Praxis profesional y realidad clínica: la construcción de la desnutrición infantil como objeto terapéutico en un centro de atención primaria en la Ciudad de Buenos Aires. Cuadernos de Antropología Social, nº.25, pp.189-207, 2007.

HERNANDO, A. Género y Sexo. Mujeres, identidad y modernidad. Claves de Razón práctica, nº.188, pp.64-88, 2008.

JENKS, C. Childhood. London: Rotledge, 1996.

JELIN, E. Pan y afectos, la transformación de las familias. Buenos Aires: Fondo de Cultura Económica, 1998.

INDEC (Instituto Nacional de Estadísticas y Censos de la República Argentina). Encuesta permanente de Hogares. Primer semestre 2013. Disponible en http://www.indec.mecon.ar/principal.asp?id_ tema $=76$. Acceso 25/3/2014.

LARA FLORES, S. M. ¿Es posible hablar de un trabajo decente en la agricultura moderno-empresarial en México? El cotidiano, Vol.23, $\mathrm{n}^{\mathrm{o}} .147$, pp. $25-33,2008$.

LEAVY, P. Soy factor de riesgo. Un estudio desde la antropología de la niñez sobre la implementación de políticas publicas sociosanitarias en el Departamento de Orán, Salta. III ${ }^{a}$ Jornadas Antropología Social del Centro. Olavarría, 5, 6 y 7 de octubre de 2011. Disponible en: http:/es.scribd.com/doc/68502601/Libro-de-Actas-III-Jornadasde-Antropologia-del-Centro-2011

LORENZETTI, M. I. Relaciones interétnicas y prácticas de atención a 
la salud en el Chaco. Corpus. Archivos virtuales de la alteridad americana, Vol.1, $\mathrm{n}^{\circ} .2,2011$, url: http://corpusarchivos.revues. $\operatorname{org} / 1150$

, M. I. Del 'capital social' a la 'culturalización de la pobreza': la construcción de alteridad en los programas de salud para la población indígena". IV ${ }^{a}$ Jornadas de Jóvenes Investigadores, Instituto de Investigaciones Gino Germani, Facultad de Cs. Sociales, Universidad de Buenos Aires. Buenos Aires, 19 al 20 de Septiembre, 2007.

M. I. Re-significaciones neoliberales de 'lo Indígena': las articulaciones de políticas de reconocimiento y políticas sociales en Argentina. 2006. Tesis (Licenciatura en Ciencias Antropológicas), Facultad de Filosofía y Letras, Universidad de Buenos Aires UBA, Buenos Aires, D.F.

MACEIRA, D. (Comp.). Atención primaria en Salud. Enfoques interdisciplinarios. Buenos Aires: Editorial Paidos, 2008.

MARCÚS, J. 'Por nuestras hijas', vínculos en las familias. In: MARGULIS, M. y Otros. Juventud, Cultura, Sexualidad: La dimensión cultural en las relaciones afectivas y sexuales de los jóvenes de Buenos Aires. Buenos Aires: Biblos, 2003, pp. 263-280.

MENÉNDEZ, E. Modelos de atención de los padecimientos: de exclusiones teóricas y articulaciones prácticas. In: SPINELLI H. (Comp.). Salud Colectiva: cultura, instituciones y subjetividad; epidemiología, gestión y políticas. Buenos Aires: Lugar Editorial, 2004.

MOORE, Henrrietta, 1988. Feminism and Anthropology. Cambridge, Polity Press.

NARI, M. Maternidad, política y feminismo. In: LOZANO, F. G.; PITA, V. y INI, G.. Historia de las mujeres en la Argentina. Siglo XX, Tomo II, Buenos Aires: FCE, 2000.

ORTALE, S. Saber médico y desnutrición en el Gran La Plata. In: BARONE, M. y SHIAVONI, L. (Comp.). La Argentina de los'90: trabajo, salud y género. Estudios en sectores medios y pobres. Posadas: Ed. de la Univ. Nac. de Misiones / Secretaría de Cultura de la Provincia. de Misiones, 2003.

PALOMAR VEREA, C. Maternidad: historia y cultura. La ventana. Revista de Estudios de Género, Vol. III, nº.22, pp.35-67, 2005. 
Perfil SANA. Perfil de la salud Ambiental de la Niñez en la Argentina. Ministerio de la Salud de la Nación, 2007.

RAMIREZ HITA, S. La contribución del dato etnográfico al registro del dato epidemiológico. Epidemiología sociocultural indígena quechua de la ciudad de Potosí. Salud Colectiva, Buenos Aires, Vol.5, n.1, pp.63-85, 2009.

RODRÍGUEZ GARCÍA, M. San Ramón de la Nueva Orán, ciudad encajonada y dispersa. Informe Agencia Extensión Rural, sede Orán, Instituto de Tecnología Agropecuaria - INTA, 2009.

ROSE, N. Identidad, genealogía, historia. In: HALL, S., y DU GAY, P. (Comps.). Cuestiones de identidad cultural. Buenos Aires: Amorrortu Editores, 2003.

SALOMON, D.; MASTRÁNGELO, A. Contribución de la antropología a la comprensión ecoepidemiológica de un brote de leishmaniasis tegumentaria americana en las "2.000 hectáreas”, Puerto Iguazú, Argentina. Rev. Argent. Salud Pública, Vol.1, n’.4, pp.06-13, 2010.

SCHEPERD-HUGHES, N. 1992. Death without Weeping. The Violence of Everyday Life in Brazil. University of California Press.

SCHEPER-HUGHES, N. \& SARGENT, C. (Eds.). Small Wars. The Cultural Politics of Childhood. Berkeley: University of California Press, 1998.

SOCÍAS, M. E.; JUAREZ, M.; CAJAL, S. P.; VILLALPANDO, C.; et al. Tratamientos comunitarios de geohelmintiasis en zonas endémicas para S. stercoralis: desarrollo de una plataforma multidisciplinaria para la evaluación de nuevas estrategias de manejo. XII ${ }^{\circ}$ Congreso de la Sociedad Argentina de Infectología, Córdoba, 16-18 de Mayo de 2012 .

SPINELLI, H. Las dimensiones del campo de la salud en Argentina. Salud Colectiva, Vol.6, nº.3, pp.275-293, 2010.

SZULC, A. P. 2006. "Antropología y niñez: de la omisión a las 'culturas infantiles"”. En: Wilde, G y P. Schamber (Eds) Cultura, comunidades y procesos contemporáneos. Editorial SB, Colección "paradigma indicial". Buenos Aires.

TORRES, M. Malnutrición y heterogeneidad socio-ambiental: Un análisis en escolares urbanos de 9 a 16 años residentes en La Plata, Argentina. Runa [online]. 2012, vol.33, n.1, pp. 85-106. ISSN 1851- 
9628.

TRONTO, J. Moral Boundaries. A political new argument for an ethic care. London: Routledge, 1993.

TARANTO N.J., CAJAL S.P., DE MARZI M.C., et al. Clinical status and parasitic infection in a Wichí Aboriginal community in Salta, Argentina. Trans R Soc Trop Med Hyg 2003; 97: 554-8.

TARDUCCI, M. Maternidades en el siglo XXI. Buenos Aires: Espacio, 2008.

TORRES ALIAGA, T. y TORRES SECCHI, A. ¿Por qué callan si nacen llorando? Poder, accesibilidad y diferencias culturales en Salud. Iruya, 1978-2008. Formosa, Argentina: Editoria Endepa, Equipo Nacional de Pastoral Indígena, 2010.

TORRES SECCHI, A. Estado, Mercado y Salud. La lucha contra la mercantilización. IX Jornadas Salud y Sociedad. Colectivo Salud y Sociedad. Por una militancia en salud. Facultad de Filosofía y Letras 10 al 13 de Julio de 2012, Ciudad de Buenos Aires.

WORLD HEALTH ORGANIZATION (WHO). Control de las helmintiasis en los países del Caribe ingles y francés: Hacia la Resolución 54.19 de la Asamblea Mundial de la Salud. [En línea] Kingstone: OPS/ OMS; 2007 [fecha de acceso 8 de julio de 2012] url: http://www. paho.org/Spanish/AD/DPC/CD/psit-sth-jamaica.htm

Recebido: 29/04/2014.

Aprovado: 15/11/2014. 